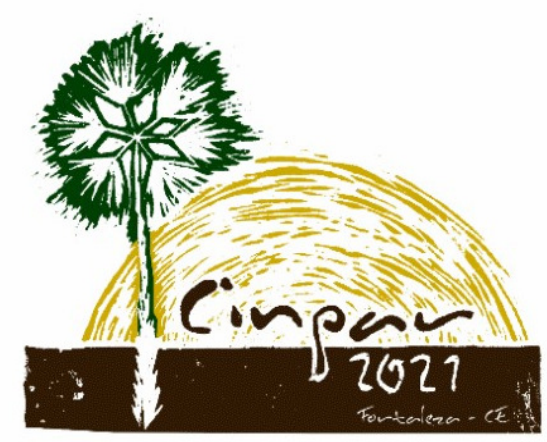

XVII Congresso Internacional sobre Patologia e

Reabilitação das Construções

XVII Congreso Internacional sobre Patología y Rehabilitación de las Construcciones

XVII International Conference on Pathology and Constructions Rehabilitation

FORTALEZA (Brasil), 3 a 5 de junho de 2021

https://doi.org/10.4322/CINPAR.2021.150

\title{
Reforço das Longarinas de uma Ponte em Concreto Armado por meio de Encamisamento em Concreto Armado
}

\section{Strengthening of Reinforced Concrete Bridge Beams with Reinforced Concrete Jacketing}

\author{
Rafaela Guimarães ${ }^{1}$, Mayra Perlingeiro², Luiz Carneiro ${ }^{3}$ \\ ${ }^{1}$ Universidade Federal Fluminense, Niterói/RJ, Brasil, rafaelaguimaraes@id.uff.br \\ 2 Universidade Federal Fluminense, Niterói/RJ, Brasil, mayraperlingeiro@id.uff.br \\ ${ }^{3}$ Universidade Federal Fluminense, Niterói/RJ, Brasil, luizcarneiro@id.uff.br
}

Resumo: Este trabalho apresenta formulações para a avaliação de capacidade de carga e de reforço estrutural por meio da técnica de encamisamento em concreto armado das longarinas de uma ponte em concreto armado, da década dos anos de 1960. A análise dos resultados permitiu avaliar a exequibilidade de método de reforço proposto, quanto aos aspectos construtivos e técnicos. $\mathrm{O}$ encamisamento em concreto armado apresenta-se como uma solução viável para o projeto de reforço.

Palavras-chave: Reforço; Longarinas; Ponte; Concreto Armado; Encamisamento.

\section{Introdução}

Nos últimos anos, os programas de ampliação e de concessões da malha rodoviária brasileira deflagrados pelo governo federal e por diversos governos estaduais mostraram problemas de ordem funcional e estrutural das pontes e viadutos que compõem esta malha.

Uma extensa vida em serviço significa a exposição aos agentes degradantes por um longo período, o que pode prejudicar o desempenho estrutural de uma obra. Para além das questões ligadas à degradação, as estruturas se figuram em períodos de evolução das atividades humanas e, como consequência, o seu modo de utilização pode passar por mudanças, comprometendo a sua funcionalidade e segurança.

Sob essa ótica, o reforço estrutural de estruturas tem sido uma alternativa à sobreposição de novas construções, permitindo que estas resistam a cargas de projeto mais elevadas, além de corrigir a perda de resistência devido à deterioração, deficiências de projeto ou de execução, assim como aumentar a ductilidade dos elementos estruturais.

O encamisamento em concreto armado é o método construtivo mais utilizado para reforço de pontes, especialmente para aquelas de pequeno porte, ou seja, para a maioria das pontes das rodovias federais do Brasil. Este artigo busca, portanto, apresentar formulações para a avaliação de capacidade de carga e de reforço estrutural por meio da técnica de encamisamento em concreto armado das longarinas de uma ponte em concreto armado.

\section{Reabilitação de Pontes em Concreto Armado}

Busca-se analisar a reabilitação estrutural de pontes devido ao aumento de carregamento por adequação da estrutura às normas vigentes e aos requisitos de segurança determinados por essas normas. Diante disso, é 
preciso o conhecimento do ano de realização do projeto e dos elementos de projeto característicos do período de construção para que seja identificada a real necessidade de seu reforço estrutural.

Entre as revisões das normas, ocorreram alterações no cálculo do coeficiente de impacto e no trem-tipo de projeto, além da consideração do recapeamento, prescrito na norma ABNT NBR 7187 (2003), o que conduz ao aumento da sobrecarga permanente.

Quanto à capacidade de carga dessas estruturas, o critério que determina a necessidade dessas intervenções é justamente a avaliação destas para as solicitações atuais de operação. Para a correta avaliação de capacidade de carga, é indispensável o conhecimento das propriedades mecânicas dos materiais que compõem a estrutura, como a resistência característica do concreto original e das armaduras.

De acordo com o Manual de Recuperação de Pontes e Viadutos (DNIT, 2010), o aço empregado nas obras mais antigas era o CA 37, atual CA 25, e, posteriormente, o aço CAT 50 com espiras deficientes e sem a consideração da fadiga. Atualmente, o aço utilizado é o CA 50 especificado na norma ABNT NBR 7480 (2007), com a consideração do fenômeno da fadiga.

O cimento CPI foi o primeiro a ser produzido no Brasil e o mais utilizado na mistura do concreto presente nas estruturas brasileiras, conforme Thomaz (2010), desde a década de 1940 até o início da década de 1990.0 cimento CPIII surgiu na década de 1960 e o cimento CPIV, na década de 1970, obtendo uma pequena participação na produção brasileira. Entretanto, desde 2010, o cimento CPII, cuja produção teve início na década de 1990, passou a ser o tipo de cimento mais produzido no país.

\section{Encamisamento em Concreto Armado}

Esta solução consiste na adição de armaduras longitudinais e transversais, que são envoltas por concreto ou argamassa, em zonas onde há insuficiência de armadura, de modo que as vigas têm novas dimensões definidas conforme a necessidade de reforço.

O apicoamento da peça é feito com o intuito de retirar a camada superficial de argamassa nas faces da viga e a camada de concreto, encontrando os estribos originais da viga. Em seguida, é feita a perfuração, para a passagem dos estribos novos, e limpeza das superfícies com jato de ar comprimido. Após a retirada de partículas soltas ou inertes, são colocadas as armaduras transversais que devem ser soldadas ou sobrepostas às originais no caso de emendas. Por último, são colocadas as armaduras longitudinais de reforço e fixadas as formas suspensas para a realização da concretagem.

Recomendações para a utilização dessa técnica são apresentadas pelo Comité Euro-Internacional du Béton (CEB) para melhorar a aderência entre os materiais e controlar retração. Estas foram compiladas do Bulletin $d^{\prime}$ Information no.162 - Assessment of concrete structures and design procedures for upgrading (redesign) de 1983 e listadas por Simões (2007):

- adicionar de uma camada com espessura mínima de $75 \mathrm{~mm}$ a $100 \mathrm{~mm}$, possibilitando o lançamento e adensamento do novo concreto e o posicionamento da nova armadura;

- saturar o concreto antigo por pelo menos seis horas antes de aplicar o concreto novo;

- evitar formação de bolhas de ar, aplicando concreto ou argamassa com uma maior fluidez e a partir de um mesmo lado da forma;

- promover a cura com umedecimento da superfície ou cobrindo-a com materiais úmidos, tais como areia, espuma, estopa e outros. Este umedecimento deve persistir por dez dias, no mínimo;

- usar concreto de resistência característica de, no mínimo, $5 \mathrm{MPa}$ a mais do que a do concreto existente.

O Comitê Europeu recomenda, ainda, limitar a tensão tangencial na superfície de ligação e que o acréscimo de área na seção transversal da viga seja mantido até um terço da área da seção original. Ademais, é indicado que o concreto ou a argamassa de reforço tenham resistência mecânica, módulo de elasticidade e coeficiente de dilatação compatíveis com os do concreto existente.

\subsection{Fundamentos de dimensionamento}

Reforço das Longarinas de uma Ponte em Concreto Armado por meio de Encamisamento em Concreto Armado 


\subsection{1 - Reforço à flexão}

Para o cálculo da resistência à flexão de uma estrutura de concreto armado reforçada com ensamisamento, são estabelecidas as seguintes considerações no Estado Limite Último (ELU):

- as deformações específicas da armadura e do concreto são diretamente proporcionais à distância da linha neutra, portanto, as seções planas permanecem planas até a ruptura;

- aderência perfeita entre aço e concreto e entre reforço e concreto;

- a deformação específica máxima para o concreto é de 3,5\%;

- a resistência do concreto à tração é desprezada.

As expressões para o dimensionamento das armaduras longitudinais complementares são obtidas a partir do diagrama retangular simplificado de tensões na seção após o reforço, ilustrado na Figura 1.

Após o reforço estrutural, a posição da linha neutra varia daquela obtida no ELU para a seção original. Dessa forma, realiza-se um procedimento iterativo, no qual fixam-se valores da armadura de reforço e de sua altura útil.

De modo alternativo, quando a verificação das áreas de aço necessárias para resistir às solicitações internas atuais for previamente realizada, pode-se estimar a altura útil da armadura total, equivalente à armadura existente e de reforço inicial, e, em seguida, fazer a correção da área de aço de reforço.

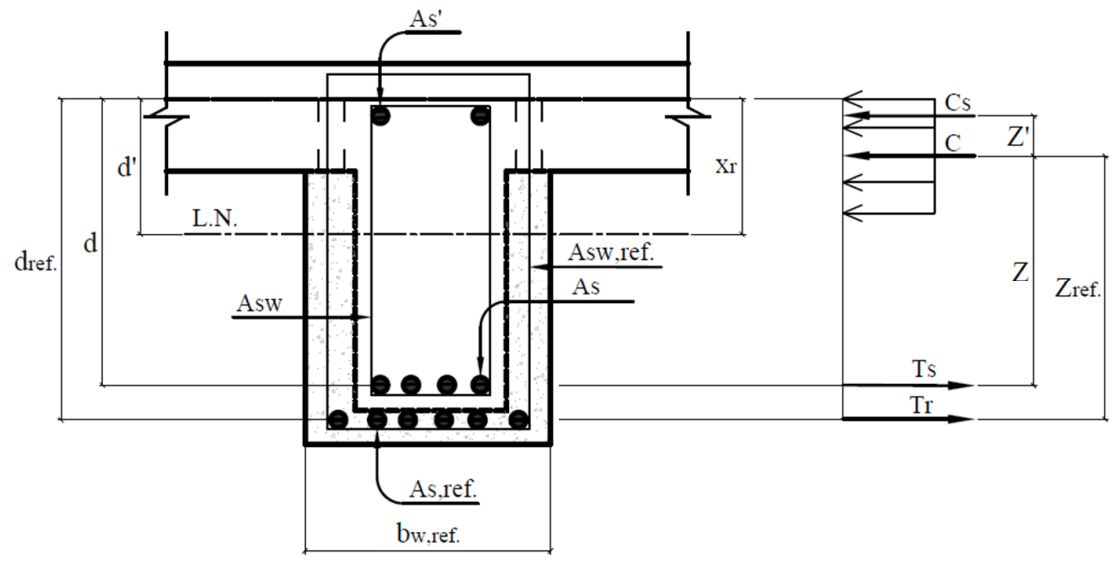

Figura 1 - Seção transversal de viga reforçada com encamisamento em concreto armado

Da Figura 1, tem-se a Equação 1, que determina a profundidade da linha neutra de uma viga retangular reforçada.

$$
0,85 \cdot f_{c d} \cdot b_{w, r e f .} \cdot x_{r}+A_{s}^{\prime} \cdot E_{s} \cdot \varepsilon^{\prime}{ }_{s}=A_{s} \cdot f_{y d}+A_{s, r e f .} \cdot f_{y d}
$$

A Equação 2 determina o momento resistente da viga reforçada.

$$
M_{r d}=A_{s} \cdot f_{y d}\left(d-0,40 x_{r}\right)+A_{s}^{\prime} \cdot E_{s} \cdot \varepsilon^{\prime}{ }_{s}\left(0,40 x_{r}-d^{\prime}\right)+A_{s, r e f .} \cdot f_{y d}\left(d_{\text {ref. }}-0,40 x_{r}\right)
$$

onde:

$A_{s}$ - área da armadura longitudinal interna de tração;

$\mathrm{A}_{\mathrm{S}}^{\prime}$ - área da armadura longitudinal interna de compressão;

$\mathrm{A}_{\mathrm{s}, \text { ref. }}$ - área da armadura longitudinal interna de reforço;

$\mathrm{f}_{\mathrm{yd}}$ - resistência de cálculo ao escoamento da armadura longitudinal interna de tração;

$\mathrm{f}_{\mathrm{cd}}$ - resistência de cálculo do concreto à compressão;

$\mathrm{x}_{\mathrm{r}}$ - profundidade da linha neutra após reforço estrutural;

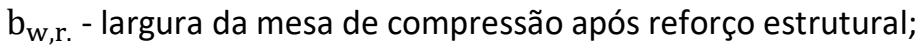

Reforço das Longarinas de uma Ponte em Concreto Armado por meio de Encamisamento em Concreto Armado 
$\mathrm{M}_{\mathrm{rd}}$ - momento resistente de cálculo;

$\mathrm{d}_{\mathrm{r}}$ - altura útil da armadura longitudinal interna de tração do reforço;

d - altura útil da armadura longitudinal interna de tração;

d' - distância entre a armadura longitudinal interna de compressão e o bordo mais comprimido.

Após o reforço estrutural, a posição da linha neutra varia daquela obtida no ELU para a seção original. Dessa forma, realiza-se um procedimento iterativo, no qual fixam-se valores da armadura de reforço e de sua altura útil.

De modo alternativo, quando a verificação das áreas de aço necessárias para resistir às solicitações internas atuais for previamente realizada, pode-se estimar a altura útil da armadura total, equivalente à armadura existente e de reforço inicial, e, em seguida, fazer a correção da área de aço de reforço utilizando a Tabela de Dimensionamento à Flexão Simples.

A armadura de reforço inicial é dada pela Equação 3.

$$
\mathrm{A}_{\mathrm{s} \text {,ref.inicial }}=\mathrm{A}_{\text {necessária }}-\mathrm{A}_{\text {existente }}
$$

A altura útil equivalente é calculada pela Equação 4.

$$
\mathrm{d}_{\text {eq. }}=\frac{\mathrm{A}_{\text {existente }} \cdot \mathrm{d}+\mathrm{A}_{\mathrm{s}, \text { ref.inicial }} \cdot \mathrm{d}_{\mathrm{r}}}{\mathrm{A}_{\text {existente }}+\mathrm{A}_{\mathrm{s}, \text { ref.inicial }}}
$$

$\mathrm{O}$ método de cálculo das áreas de aço adota os parâmetros adimensionais $\mathrm{k}_{\mathrm{z}}, \mathrm{k}_{\mathrm{x}}, \mathrm{e} \mathrm{k}_{\mathrm{md}}$. Uma vez obtida a área de aço longitudinal complementar, devem-se utilizar barras de diâmetro comercial e fazer o detalhamento das armaduras observando-se os requisitos da norma ABNT NBR:6118 (2014).

Nessa técnica, é importante atentar para a tensão de aderência na superfície de ligação entre os concretos de diferentes idades. Para evitar a ruína por cisalhamento nessa ligação, recomenda-se limitar os valores da tensão cisalhamento da interface de ligação.

O código CEB (1993) sugere que a tensão de cisalhamento média (v. Equação 5) nas interfaces seja inferior ao valor da resistência de aderência, devida à coesão. Essa resistência é igual a resistência à tração de cálculo do concreto $\left(f_{\text {ctd }}\right)$ multiplicada por um fator $\beta$ que depende da superfície da junta, sendo igual a 0,2 para superfícies lisas e 0,4 para superfícies rugosas.

$$
\tau=\beta \cdot \mathrm{f}_{\mathrm{ctd}}
$$

Por fim, faz-se a verificação à fadiga da armadura de flexão no ELS, prescrita na norma ABNT NBR:6118 (2014) e descrita no item 5.2.2.

\subsection{2 - Reforço à força cortante}

O dimensionamento do reforço estrutural com estribos, assim como no dimensionamento à flexão, é realizado no Estado Limite Último (ELU). O cálculo da armadura transversal baseia-se no modelo de Treliça de Mörsch, associado aos mecanismos resistentes complementares desenvolvidos no interior do elemento estrutural e traduzidos pela componente $\left(\mathrm{V}_{\mathrm{c}}\right)$.

A resistência de cálculo de vigas de concreto reforçadas pode ser determinada adicionando a contribuição proveniente da armadura transversal de reforço $\left(V_{\text {ref. }}\right)$ às parcelas da armadura transversal existente $\left(\mathrm{V}_{\mathrm{sw}}\right) \mathrm{e}$ do concreto $\left(\mathrm{V}_{\mathrm{c}}\right)$.

A força cortante resistente de cálculo é determinada pela Equação 6 .

$$
\mathrm{V}_{\mathrm{Rd}}=\mathrm{V}_{\mathrm{sw}}+\mathrm{V}_{\mathrm{c}}+\mathrm{V}_{\text {ref. }}
$$

onde:

$\mathrm{V}_{\mathrm{SW}}$ - parcela da força cortante resistida pela armadura transversal;

$\mathrm{V}_{\mathrm{c}}$ - parcela de força cortante absorvida por mecanismos complementares ao da treliça;

$\mathrm{V}_{\text {ref. }}$ - parcela da força cortante resistida pela armadura transversal de reforço.

Reforço das Longarinas de uma Ponte em Concreto Armado por meio de Encamisamento em Concreto Armado 
Adota-se o modelo de cálculo I, descrito na norma ABNT NBR:6118 (2014). Esse modelo admite diagonais de compressão inclinadas a $45^{\circ} \mathrm{em}$ relação ao eixo longitudinal do elemento estrutural e parcela complementar $\left(V_{c}\right)$ com valor constante, independente da força cortante de dimensionamento.

Conhecida a área de aço transversal existente, determina-se pela Equação 7 a parcela de força cortante resistida por ela:

$$
\mathrm{V}_{\mathrm{sw}}=\left(\frac{\mathrm{A}_{\mathrm{sw}}}{\mathrm{s}}\right) 0,9 \mathrm{~d} \cdot \mathrm{f}_{\mathrm{yd}}
$$

Então, igualando a força cortante resistente de cálculo com a solicitante de cálculo, obtém-se a parcela que deve ser resistida pela armadura transversal complementar. As duas condições a seguir devem ser satisfeitas com o uso das Equações 8 e 9.

$$
\begin{gathered}
\mathrm{V}_{\mathrm{sd}} \leq \mathrm{V}_{\mathrm{rd} 2} \\
\mathrm{~V}_{\mathrm{sd}} \leq \mathrm{V}_{\mathrm{rd} 3}=\mathrm{V}_{\mathrm{c}}+\mathrm{V}_{\mathrm{sw}}
\end{gathered}
$$

onde:

$\mathrm{V}_{\text {sd }}$ - força cortante solicitante de cálculo na seção;

$\mathrm{V}_{\mathrm{rd2}}$ - força cortante resistente de cálculo relativa à ruína das diagonais comprimidas de concreto;

$\mathrm{V}_{\mathrm{rd3}}$ - força cortante resistente de cálculo relativa à ruína por tração diagonal.

A área de aço transversal de reforço é determinada por uma taxa em $\mathrm{cm}^{2} / \mathrm{m}$, obtida pela Equação 10 .

$$
\frac{A_{\text {sw,ref. }}}{s}=\frac{V_{\text {ref. }}}{0,9 d_{\text {ref. }} \cdot f_{\text {yd }}}
$$

onde:

$\mathrm{V}_{\text {ref. }}$ - parcela da força cortante resistida pela armadura transversal complementar;

$\mathrm{d}_{\text {ref. }}$ - altura útil da viga reforçada;

$\mathrm{f}_{\mathrm{yd}}$ - tensão de escoamento do aço.

Por fim, faz-se a verificação à fadiga da armadura transversal no ELS, prescrita na norma ABNT NBR:6118 (2014) e descrita no item 3.1.4.

\section{Estudo de caso}

A estrutura original consiste em uma ponte em concreto armado, constituída por um vão de $20,0 \mathrm{~m}$ finalizados em balanços de 4,5 m cada, somando um total de $29,0 \mathrm{~m}$. As transversinas de apoio e intermediárias são tradicionalmente ligadas às lajes e espaçadas em $5,0 \mathrm{~m}$, como ilustrado na Figura 2. A Figura 3 ilustra o corte transversal no meio de vão da ponte existente, que corresponde à seção transversal típica do período dos anos de 1960 a 1975. Quanto aos materiais originais da ponte, o concreto apresenta resistência característica à compressão de $22 \mathrm{MPa}$ e o aço possui resistência característica ao escoamento de $500 \mathrm{MPa}$.

Para a adequação ao gabarito transversal atual e garantia da segurança dos usuários, é feita a substituição dos guarda-rodas e guarda-corpos existentes por barreiras tipo New Jersey. Além disso, são adicionadas lajes de transição de 4,0 m, seguindo o comprimento da aba existente. A seção transversal da ponte reabilitada é ilustrada na Figura 4.

Para a determinação dos carregamentos permanentes nas vigas principais de ambos os projetos foram utilizados métodos analíticos clássicos. A definição dos carregamentos devidos às cargas móveis foi feita conforme a ABNT NB-6 (1960) para a ponte original e, posteriormente, segundo a ABNT NBR 7188 (2013) para a ponte reabilitada. Os dimensionamentos à flexão e ao cisalhamento das longarinas seguiram as

Reforço das Longarinas de uma Ponte em Concreto Armado por meio de Encamisamento em Concreto Armado 
diretrizes da ABNT NB-1 (1960) para projeto da década de 1960 e da ABNT NBR 6118 (2014) para o projeto de reabilitação.

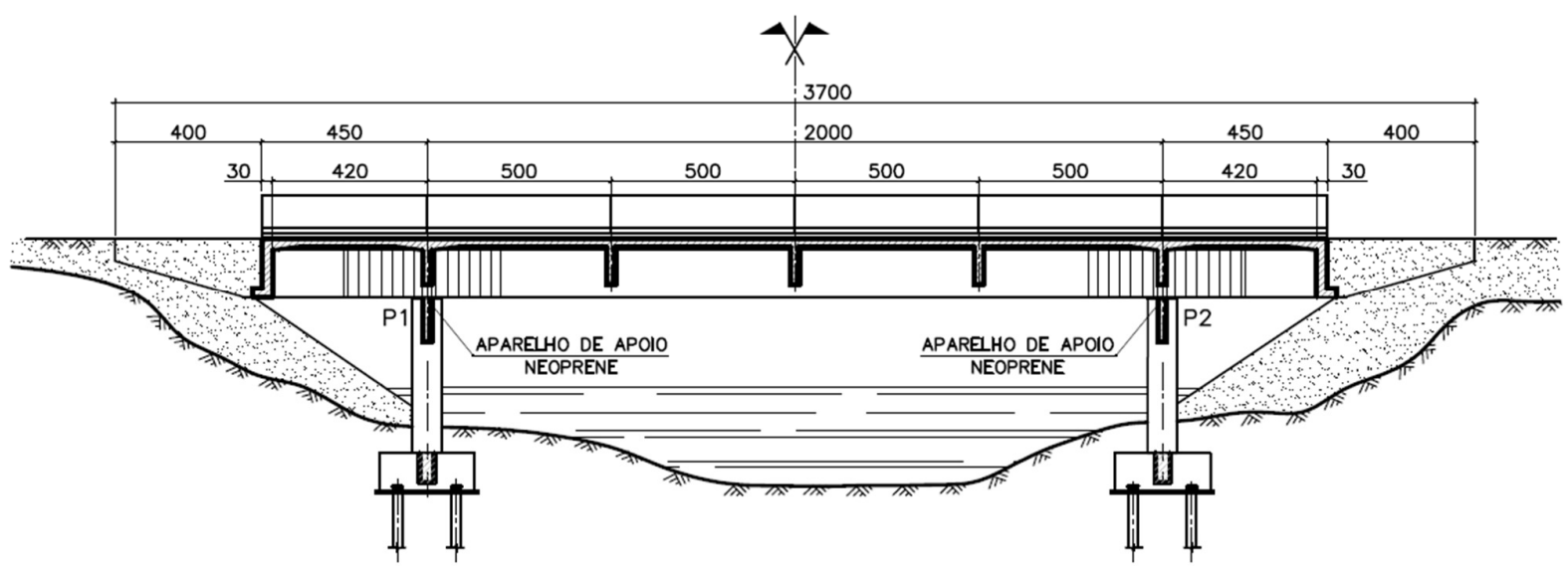

Figura 2 - Meio corte da elevação longitudinal (cotas em cm)

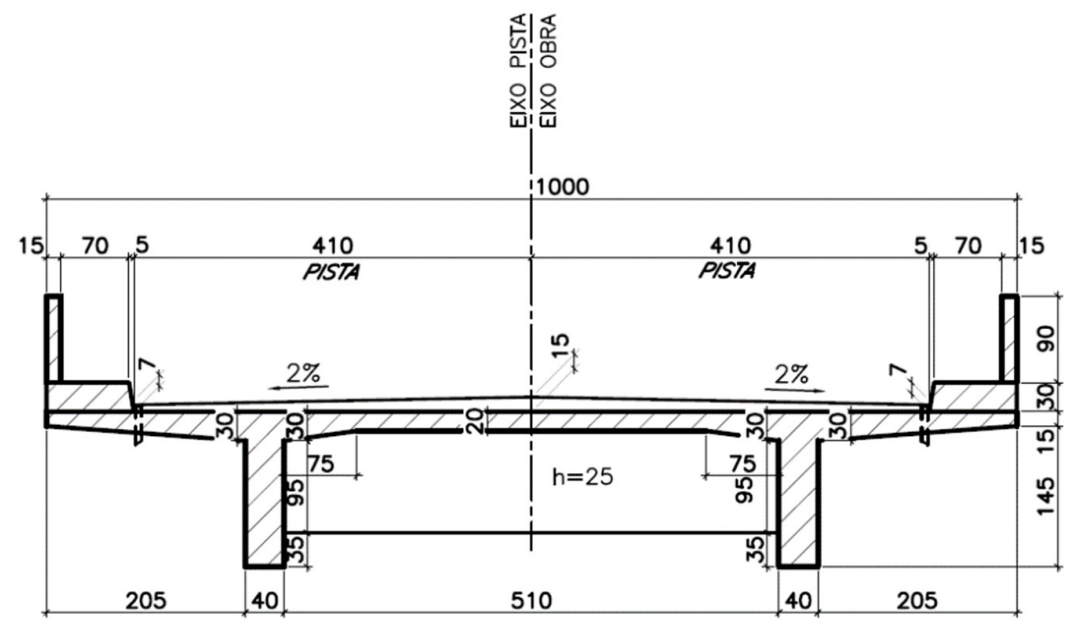

Figura 3 - Corte transversal no meio de vão da ponte existente (cotas em cm)

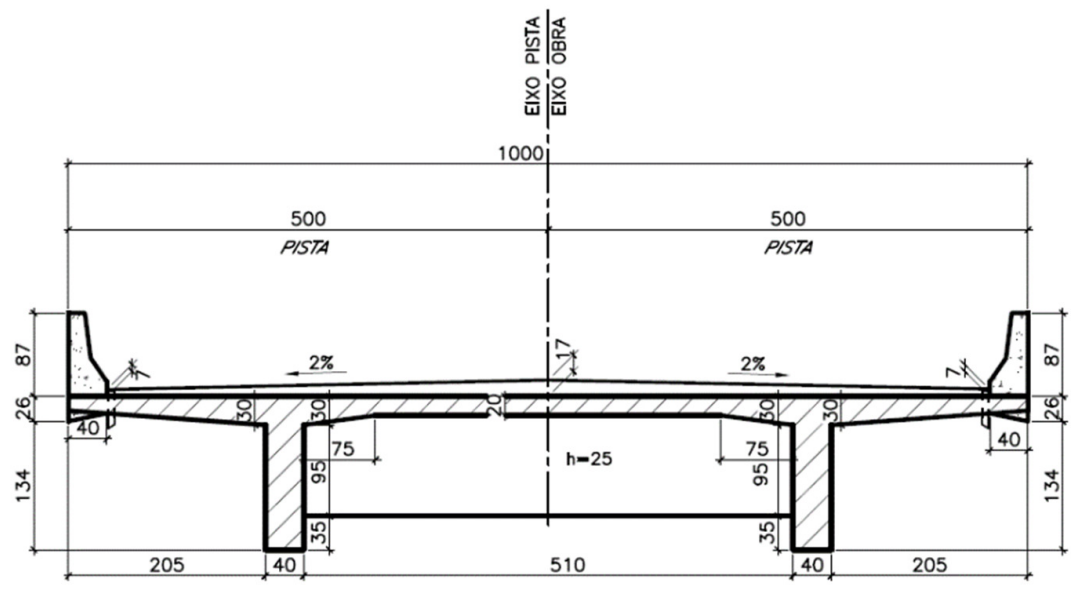

Figura 4 - Corte transversal no meio de vão da ponte reabilitada (cotas em $\mathrm{cm}$ ) 
Conforme a ABNT NB-1 (1960), as vigas sob a ação de solicitações de flexão simples ou composta são dimensionadas em função da carga de ruptura (Estádio III), com seus respectivos coeficientes de segurança ou pelas tensões admissíveis (Estádio II), sem os efeitos de fadiga das armaduras. O dimensionamento das armaduras de flexão e transversais da ponte existente é feito no Estado Limite Último em Guimarães (2019) considerando-se que o concreto adquiriu resistência ao longo de 54 anos, tendo, atualmente, resistência característica à compressão de $28 \mathrm{MPa}$.

De acordo com a ABNT NB-2 (1961), os coeficientes de segurança no Estádio III, para peças solicitadas à flexão simples ou composta, são iguais a 2,00 para carga móvel, definidos nas normas ABNT NB-6 (1960) e ABNT NB-7 (1943) e 1,65 para cargas permanentes. Esses coeficientes foram alterados entre as normas ABNT NB2 (1961) e ABNT NBR 8681 (2004), passando de 2,00 e 1,65 para 1,50, para ações variáveis,e 1,35, para ações permanentes (ações desfavoráveis), respectivamente.

O resultado do dimensionamento ao momento de flexão de ambas as pontes é apresentado no Quadro $1 \mathrm{e}$ do dimensionamento à força cortante no Quadro 2. Ao comparar os resultados das armaduras existentes na ponte original com os resultados das armaduras necessárias para o caso da ponte reabilitada, verifica-se que há deficiência de armadura longitudinal e transversal. Portanto, constata-se a necessidade de reforço estrutural das vigas principais nas seções de cálculo.

Quadro 1 - Resultado do dimensionamento ao momento de flexão das pontes na seção $S_{5}$

\begin{tabular}{|c|c|c|c|c|c|c|c|c|}
\hline Seção & \multicolumn{5}{|c|}{ Momento de Flexão Característico } & \multicolumn{2}{|c|}{$\begin{array}{l}\text { Momento de Flexão } \\
\text { de Dimensionamento }\end{array}$} & \multirow{2}{*}{$\begin{array}{c}\text { Armadura } \\
\text { Inferior }\end{array}$} \\
\hline $\mathrm{S}_{5}$ & $\begin{array}{c}\mathrm{Mg}_{\mathrm{g} 1} \\
(\mathrm{kN} . \mathrm{m})\end{array}$ & \multicolumn{2}{|c|}{$\begin{array}{c}\mathrm{Mg2} \\
(\mathrm{kN} \cdot \mathrm{m})\end{array}$} & $\begin{array}{c}\mathrm{M}_{\mathrm{q} 1} \\
(\mathrm{kN} \cdot \mathrm{m})\end{array}$ & $\begin{array}{c}\mathrm{M}_{\mathrm{q} 2} \\
(\mathrm{kN} \cdot \mathrm{m})\end{array}$ & $\begin{array}{l}\text { Msd,máx. } \\
\text { (kN.m) }\end{array}$ & $\begin{array}{l}\text { Msd,mín. } \\
\text { (kN.m) }\end{array}$ & \\
\hline Ponte Original & 1.371 & \multicolumn{2}{|c|}{847} & 2.660 & -780 & 8.980 & 657 & 129 \\
\hline Ponte Reabilitada & 1.305 & $1.004 *$ & $217^{* *}$ & 3.899 & -1.490 & 8.967 & -713 & 129 \\
\hline
\end{tabular}

* Valores máximos da envoltória; ** Valores mínimos da envoltória.

Quadro 2 - Resultado do dimensionamento à força cortante das pontes na seção So,d

\begin{tabular}{|c|c|c|c|c|c|c|}
\hline Seção & \multicolumn{4}{|c|}{ Força Cortante Característica } & Força Cortante de & Armadura \\
\hline$S_{0, d}$ & $\mathrm{~V}_{\mathrm{g} 1}(\mathrm{kN})$ & $\mathrm{V}_{\mathrm{g} 2}(\mathrm{kN})$ & $\mathrm{V}_{\mathrm{q} 1}(\mathrm{kN})$ & $\mathrm{V}_{\mathrm{q} 2}(\mathrm{kN})$ & $\mathrm{V}_{\mathrm{sd}}(\mathrm{kN})$ & $A_{s w}\left(\mathrm{~cm}^{2} / \mathrm{m}\right)$ \\
\hline Ponte Original & 452 & 212 & 553 & $-65,4$ & 2.202 & 16,8 \\
\hline Ponte Reabilitada & 451 & 278 & 1.098 & -156 & 2.630 & 56,5 \\
\hline
\end{tabular}

A espessura do concreto novo é definida de modo que seja possível posicionar a armadura complementar longitudinal e transversal. Com objetivo de melhorar a aderência entre os materiais e controlar retração, 0 Bulletin 162 do CEB (1983) recomenda uma espessura mínima de 7,5 cm.

O cobrimento das longarinas da ponte original é avaliado em $2,5 \mathrm{~cm}$ e das lajes em 2,0 cm. Para que seja possível posicionar as barras de aço de reforço, adota-se o aumento da espessura da laje em $5,5 \mathrm{~cm}$, somando-se 7,5 cm de concreto novo. No vão, aumenta-se a altura da viga em $5,0 \mathrm{~cm}$ em sua face inferior. A nova altura da longarina é de $166 \mathrm{~cm}$ nos apoios e de $171 \mathrm{~cm}$ no meio de vão.

$O$ alargamento das vigas é determinado em $5,0 \mathrm{~cm}$ em cada face lateral para a extensão total da ponte, sendo a camada total de concreto novo de $7,5 \mathrm{~cm}$, conforme ilustram as Figuras 5 e 6 .

O Quadro 3 indica o resultado do dimensionamento à flexão no meio de vão com as novas propriedades geométricas da viga após o aumento da seção.

O Quadro 4 resume o resultado do dimensionamento à força cortante à direita do apoio da ponte após o encamisamento em concreto armado.

Reforço das Longarinas de uma Ponte em Concreto Armado por meio de Encamisamento em Concreto Armado 


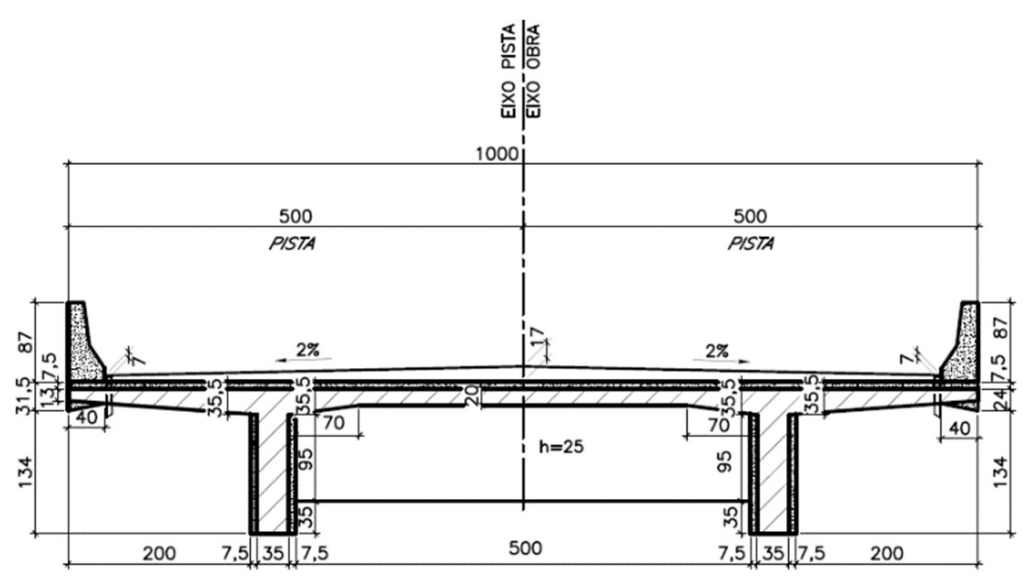

Figura 5 - Seção transversal no meio de vão da ponte com encamisamento em CA (cotas em cm)

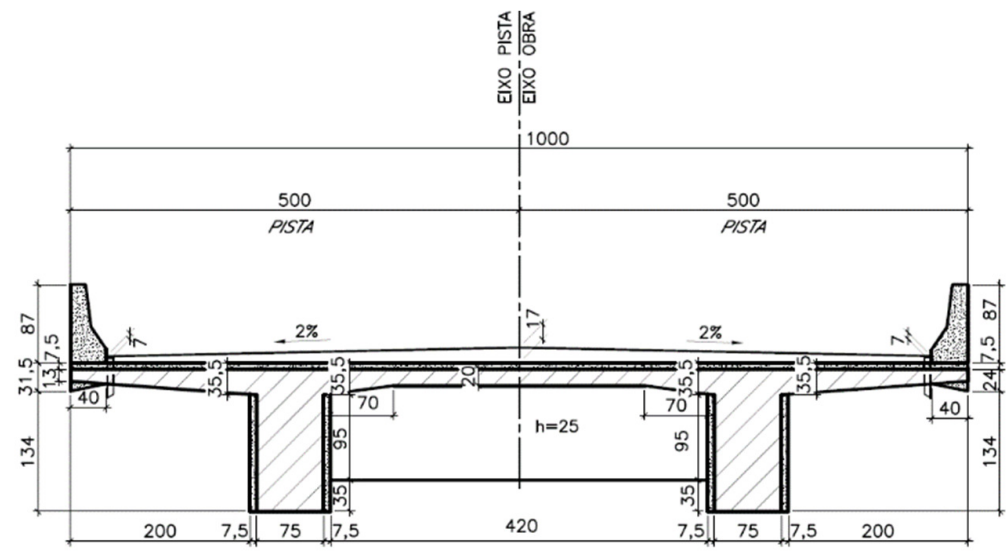

Figura 6 - Seção transversal no apoio da ponte reforçada com encamisamento em CA (cotas em cm)

Quadro 3 - Dimensionamento ao momento de flexão da ponte reabilitada com encamisamento em CA

\begin{tabular}{|c|c|c|c|c|c|c|c|c|}
\hline Seção & \multicolumn{5}{|c|}{ Momento de Flexão Característico } & \multicolumn{2}{|c|}{$\begin{array}{l}\text { Momento de Flexão } \\
\text { de Dimensionamento }\end{array}$} & $\begin{array}{c}\text { Armadura Inferior } \\
\text { Necessária }\end{array}$ \\
\hline $\mathrm{S}_{5}$ & $\begin{array}{c}\mathrm{M}_{\mathrm{g} 1} \\
(\mathrm{kN} . \mathrm{m})\end{array}$ & $\mathrm{M}_{\mathrm{g} 2}($ & N.m) & $\begin{array}{c}\mathrm{M}_{\mathrm{q} 1} \\
(\mathrm{kN} \cdot \mathrm{m})\end{array}$ & $\begin{array}{c}\mathrm{M}_{\mathrm{q} 2} \\
\text { (kN.m) }\end{array}$ & $\begin{array}{l}\text { Msd,máx. } \\
\text { (kN.m) }\end{array}$ & $\begin{array}{l}\text { Msd,mín. } \\
\text { (kN.m) }\end{array}$ & $A_{s, \text { inf. }}\left(\mathrm{cm}^{2}\right)$ \\
\hline Ponte Reabilitada & 1.749 & $1004 *$ & $217^{* *}$ & 3.899 & -1.490 & 9.566 & -713 & 152 \\
\hline
\end{tabular}

* Valores máximos da envoltória; ** Valores mínimos da envoltória

Quadro 4 - Dimensionamento à força cortante da ponte reabilitada com encamisamento em CA

\begin{tabular}{|c|c|c|c|c|c|c|c|}
\hline Seção & \multicolumn{4}{|c|}{ Força Cortante Característica } & \multicolumn{2}{c|}{$\begin{array}{c}\text { Força Cortante de } \\
\text { Dimensionamento }\end{array}$} & $\begin{array}{c}\text { Armadura } \\
\text { Transversal } \\
\text { Necessária }\end{array}$ \\
\hline $\mathrm{S}_{0, \mathrm{~d}}$ & $\mathrm{~V}_{\mathrm{g} 1}(\mathrm{kN})$ & $\mathrm{V}_{\mathrm{g} 1}(\mathrm{kN})$ & $\mathrm{V}_{\mathrm{q} 1}(\mathrm{kN})$ & $\mathrm{V}_{\mathrm{q} 1}(\mathrm{kN})$ & $\mathrm{V}_{\mathrm{sd} \text {,máx. }}(\mathrm{kN})$ & $\mathrm{V}_{\mathrm{sd}, \text { mín. }}(\mathrm{kN})$ & $\mathrm{A}_{\mathrm{sw}}\left(\mathrm{cm}^{2} / \mathrm{m}\right)$ \\
\hline $\begin{array}{c}\text { Ponte } \\
\text { Reabilitada }\end{array}$ & 568 & 278 & 1.098 & -156 & 2.789 & 611 & 54,5 \\
\hline
\end{tabular}

Reforço das Longarinas de uma Ponte em Concreto Armado por meio de Encamisamento em Concreto Armado 


\subsection{3 - Reforço à flexão}

As propriedades geométricas da seção original são:

$$
\begin{gathered}
\mathrm{d}^{\prime}=20,0 \mathrm{~cm} \\
\mathrm{~d}=151 \mathrm{~cm} \\
\mathrm{~b}_{\mathrm{w}, \text { ref. }}=90,0 \mathrm{~cm} \\
\mathrm{~b}_{\mathrm{f}, \text { ref. }}=325 \mathrm{~cm}
\end{gathered}
$$

Conforme o Quadro 1, as armaduras longitudinais existentes na seção $S_{0}$ são iguais a:

$$
\begin{aligned}
\mathrm{A}_{\mathrm{s}} & =129 \mathrm{~cm}^{2} \\
\mathrm{~A}_{\mathrm{s}}{ }_{\mathrm{s}} & =2,45 \mathrm{~cm}^{2}
\end{aligned}
$$

As armaduras necessárias para resistir o carregamento atual são apresentadas no Quadro 3 e iguais a:

$$
\begin{aligned}
A_{s} & =152 \mathrm{~cm}^{2} \\
A^{\prime}{ }_{s} & =2,45 \mathrm{~cm}^{2}
\end{aligned}
$$

A armadura superior de reforço inicial é dada pela Equação 3.

$$
\mathrm{A}_{\mathrm{s}, \text { ref.inicial }}=152-129=23,3 \mathrm{~cm}^{2}
$$

Inicialmente, considera-se a distribuição da armadura longitudinal complementar em uma camada. Sendo o cobrimento da laje de $2,0 \mathrm{~cm}$, a espessura adicional inferior de 5,0 cm e adotando barras de $20 \mathrm{~mm}$ de diâmetro, a altura útil da armadura complementar superior é igual a:

$$
\mathrm{d}_{\text {ref. }}=160+5,0-2,0+\frac{1,25}{2}=164 \mathrm{~cm}
$$

A altura útil equivalente é calculada pela Equação 4.

$$
\mathrm{d}_{\text {eq. }}=\frac{129 \times 155+23,3 \times 164}{152}=153 \mathrm{~cm}
$$

A partir do Quadro 3, tem-se o momento de flexão de dimensionamento da seção So:

$$
\mathrm{M}_{\mathrm{sd}}=9.566 \mathrm{kN} . \mathrm{m}
$$

Os parâmetros adimensionais da Tabela de Dimensionamento à Flexão Simples são determinados pelas expressões a seguir.

$$
\begin{gathered}
k_{m d}=\frac{|9.566|}{0,90 \times(1,53)^{2} \times \frac{28.000}{1,4}}=0,063 \\
k_{x}=\frac{1-\sqrt{1-\frac{2 \times 0,063}{0,85}}}{0,80}=0,097<0,450 \\
\mathrm{k}_{\mathrm{z}}=1-0,40 \times 0,097=0,961
\end{gathered}
$$

A área de aço longitudinal inferior total necessária é igual a:

$$
A_{\text {s,sup. }}=\frac{|9.566|}{0,961 \times 1,53 \times \frac{50}{1,15}}=79,2 \mathrm{~cm}^{2}
$$

Como os fatores de fadiga da armadura longitudinal não levaram à majoração das áreas de aço no dimensionamento da ponte encamisada, à não ser em 7\% na seção S2 (v. Quadro 4) não é feita uma nova verificação à fadiga.

Logo, a área de aço superior de reforço é igual a:

Reforço das Longarinas de uma Ponte em Concreto Armado por meio de Encamisamento em Concreto Armado 


$$
\mathrm{A}_{\mathrm{s}, \text { ref. }}=150-129=21,0 \mathrm{~cm}^{2}
$$

O resumo do dimensionamento do reforço à flexão é apresentado no Quadro 5.

Quadro 5 - Dimensionamento do reforço ao momento de flexão positivo no meio de vão

\begin{tabular}{|c|c|c|c|c|c|c|}
\hline $\begin{array}{c}\text { Momento de } \\
\text { Flexão de } \\
\text { Dimensionamento }\end{array}$ & $\begin{array}{l}\text { Largura da } \\
\text { Mesa de } \\
\text { Compressão }\end{array}$ & $\begin{array}{l}\text { Altura da } \\
\text { Viga }\end{array}$ & $\begin{array}{c}\text { Altura Útil da } \\
\text { Armadura }\end{array}$ & $\begin{array}{l}\text { Diâmetro } \\
\text { da barra } \\
\text { (cm) }\end{array}$ & $\begin{array}{c}\text { Altura Útil } \\
\text { da } \\
\text { Armadura } \\
\text { de Reforço }\end{array}$ & $\begin{array}{c}\text { Altura Útil } \\
\text { Efetiva }\end{array}$ \\
\hline $\mathrm{M}_{\mathrm{sd}}$ (kN.m) & $b_{f}(\mathrm{~cm})$ & $\mathrm{h}(\mathrm{cm})$ & $\mathrm{d}_{\mathrm{s}}(\mathrm{cm})$ & $\varphi(\mathrm{cm})$ & $\mathrm{d}_{\text {ref. }}(\mathrm{cm})$ & $\mathrm{d}_{\text {ef. }}(\mathrm{cm})$ \\
\hline 9.566 & 325 & 171 & 151 & 2,0 & 164 & 153 \\
\hline $\begin{array}{l}\text { Área de Aço } \\
\text { Existente }\end{array}$ & $\begin{array}{l}\text { Área de Aço } \\
\text { Necessária }\end{array}$ & $\begin{array}{c}\text { Área } \\
\text { Adicional } \\
\text { Necessária }\end{array}$ & $\begin{array}{c}\text { Parâmetro } \\
\text { Adimensional } \\
\text { da TDFS }\end{array}$ & $\begin{array}{l}\text { Área de } \\
\text { Aço Inferior } \\
\text { Corrigida }\end{array}$ & $\begin{array}{l}\text { Área de Aço } \\
\text { do Reforço }\end{array}$ & $\begin{array}{c}\text { Número de } \\
\text { Barras }\end{array}$ \\
\hline$A_{s, \text { existente }}\left(\mathrm{cm}^{2}\right)$ & $A_{s, \text { nec. }}\left(\mathrm{cm}^{2}\right)$ & $\Delta \mathrm{A}_{\mathrm{s}}\left(\mathrm{cm}^{2}\right)$ & $\mathrm{k}_{\mathrm{z}}$ & $A_{s, \text { inf. }}\left(\mathrm{cm}^{2}\right)$ & $A_{s, \text { reforço }}\left(\mathrm{cm}^{2}\right)$ & $\mathrm{n}$ \\
\hline 129 & 152 & 23,3 & 0,961 & 150 & 21,0 & 7 \\
\hline
\end{tabular}

\subsection{4 - Reforço à força cortante}

As propriedades geométricas da seção So à direita do apoio são:

$$
\begin{gathered}
\mathrm{d}=145 \mathrm{~cm} \\
\mathrm{~d}_{\text {ref. }}=151 \mathrm{~cm} \\
\mathrm{~b}_{\mathrm{w}}=80,0 \mathrm{~cm} \\
\mathrm{~b}_{\mathrm{w}, \text { ref. }}=90,0 \mathrm{~cm} \\
\mathrm{~b}_{\mathrm{f}}=275 \mathrm{~cm} \\
\mathrm{~h}_{\mathrm{f}}=22,3 \mathrm{~cm}
\end{gathered}
$$

Conforme o Quadro 4, a armadura transversal existente na seção $S_{0}$ à direita do apoio é igual a:

$$
\frac{\mathrm{A}_{\text {sw,existente. }}}{\mathrm{s}}=16,8 \mathrm{~cm}^{2} / \mathrm{m}
$$

A partir da área de aço transversal, determina-se a parcela da força cortante resistida por ela pela Equação 7:

$$
\mathrm{V}_{\mathrm{sw}}=0,9 \times 1,45 \times \frac{50}{1,15} \times 16,8=954 \mathrm{kN}
$$

A força cortante de dimensionamento para a seção So,d é obtida no Quadro 4:

$$
\mathrm{V}_{\mathrm{sd}}=2.789 \mathrm{kN}
$$

A parcela da força cortante absorvida pelo concreto $\left(V_{c}\right)$ e a força cortante resistente de cálculo relativa à ruína das diagonais comprimidas de concreto $\left(\mathrm{V}_{\mathrm{Rd} 2}\right)$ devem ser recalculadas devido ao aumento da seção transversal.

É verificada a biela de compressão:

$$
\begin{gathered}
\mathrm{V}_{\mathrm{rd}, 2}=0,27 \alpha_{\mathrm{v}} \cdot \mathrm{f}_{\mathrm{cd}} \cdot \mathrm{b}_{\mathrm{w}} \cdot \mathrm{d} \\
\mathrm{V}_{\mathrm{rd}, 2}=0,27 \times 0,888 \times \frac{28,0}{1,4} \times 0,90 \times 1,51 \times 10^{3}=3.607 \mathrm{kN} \\
\mathrm{V}_{\mathrm{sd}}=2.789 \mathrm{kN}<3.607 \mathrm{kN} \rightarrow \mathrm{Ok} !
\end{gathered}
$$

Reforço das Longarinas de uma Ponte em Concreto Armado por meio de Encamisamento em Concreto Armado 
A parcela de cortante absorvido por mecanismos complementares:

$$
\begin{gathered}
\mathrm{V}_{\mathrm{c}}=0,6 \cdot \mathrm{f}_{\mathrm{ctd}} \cdot \mathrm{b}_{\mathrm{w}} \cdot \mathrm{d} \\
\mathrm{V}_{\mathrm{c}}=0,6 \times \frac{0,7 \times 0,3 \times 28,0^{2 / 3}}{1,40} \times 0,90 \times 1,51 \times 10^{3}=1.124 \mathrm{kN}
\end{gathered}
$$

A força cortante, devido à parcela de resistência da armadura complementar transversal, é determinada por meio da Equação 6, igualando a força cortante resistente de cálculo com a solicitante de cálculo:

$$
\begin{gathered}
2.789=954+1.154+\mathrm{V}_{\text {ref. }} \\
\mathrm{V}_{\text {ref. }}=711 \mathrm{kN}
\end{gathered}
$$

A área de aço transversal de reforço é obtida pela Equação 10:

$$
\frac{A_{\text {sw,ref. }}}{\mathrm{s}}=\frac{711}{0,9 \times 1,51 \times \frac{50}{1,15}}=12,1 \mathrm{~cm}^{2} / \mathrm{m}
$$

Assim, a área de aço transversal total é igual a:

$$
\frac{A_{\text {sw,total }}}{\mathrm{s}}=16,8+12,1=28,9 \mathrm{~cm}^{2} / \mathrm{m}
$$

Em seguida, é feita a verificação à fadiga da armadura transversal. As forças cortantes de serviço máximo e mínimo na seção $S_{0, d}$ são iguais a:

$$
\begin{aligned}
& \mathrm{V}_{\text {serv. }}^{\text {máx. }}=1.395 \mathrm{kN} \\
& \mathrm{V}_{\text {serv. }}^{\text {mín. }}=768 \mathrm{kN}
\end{aligned}
$$

A tensão máxima e a tensão mínima nas armaduras de transversais são:

$$
\begin{aligned}
\sigma_{\mathrm{s}, \mathrm{w} 1} & =\frac{1.395-0,5 \times 1.124}{28,9 \times 0,9 \times 1,51} \times 10=213 \mathrm{MP} \text { ? } \\
\sigma_{\mathrm{s}, \mathrm{w} 2} & =\frac{768-0,5 \times 1.124}{26,3 \times 0,9 \times 1,51} \times 10=52,6 \mathrm{MP} \text { ? }
\end{aligned}
$$

Conforme a seção 23 da norma ABNT NBR:6118 (2014), $\Delta \mathrm{f}_{\text {sd,fad. }}=85,0$ MP ? para estribos. Portanto, o fator de fadiga é igual a:

$$
\mathrm{ff}=\frac{(213-52,6)}{85,0}=1,885
$$

A área de aço transversal final na seção $\mathrm{S}_{0, \mathrm{~d}}$ é igual a:

$$
\frac{A_{\text {sw }}}{\mathrm{s}}=28,9 \times 1,885=54,5 \mathrm{~cm}^{2} / \mathrm{m}
$$

Logo, a área total de reforço é igual a:

$$
\frac{A_{\text {sw,ref. }}}{\mathrm{s}}=54,5-16,8=37,7 \mathrm{~cm}^{2} / \mathrm{m}
$$

Reforço das Longarinas de uma Ponte em Concreto Armado por meio de Encamisamento em Concreto Armado 
O resumo do dimensionamento do reforço à força cortate é apresentado no Quadro 6.

Quadro 6 - Dimensionamento do reforço à força cortante à direita do apoio da ponte reabilitada

\begin{tabular}{|c|c|c|c|c|c|c|c|c|}
\hline \multirow{2}{*}{$\begin{array}{c}\text { Força Cortante de } \\
\text { Dimensionamento } \\
V_{s d}(k N)\end{array}$} & \multirow{2}{*}{$\begin{array}{c}\text { Área de } \\
\text { Aço de } \\
\text { Reforço } \\
\text { Calculada }\end{array}$} & \multicolumn{3}{|c|}{ Forças Cortantes Resistentes } & \multicolumn{3}{|c|}{$\begin{array}{l}\text { Forças Cortantes } \\
\text { Resistentes }\end{array}$} & \multirow{2}{*}{ 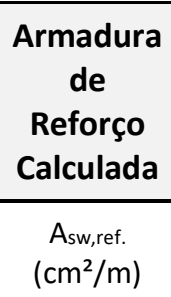 } \\
\hline & & $\mathrm{d}_{\mathrm{i}}(\mathrm{cm})$ & $d_{f}(\mathrm{~cm})$ & $b_{w}(\mathrm{~cm})$ & $\begin{array}{l}V_{\mathrm{sw}} \\
(\mathrm{kN})\end{array}$ & $\mathrm{V}_{\mathrm{c}}(\mathrm{kN})$ & $\mathrm{V}_{\text {ref. }}(\mathrm{kN})$ & \\
\hline 2.789 & 16,8 & 145 & 151 & 90 & 954 & 1.124 & 711 & 12,1 \\
\hline \multicolumn{2}{|c|}{ Força Cortante em Serviço } & \multicolumn{2}{|c|}{$\begin{array}{l}\text { Tensão na } \\
\text { Armadura } \\
\text { Transversal }\end{array}$} & $\begin{array}{c}\text { Variação } \\
\text { de } \\
\text { Tensão }\end{array}$ & $\begin{array}{c}\text { Fator } \\
\text { de } \\
\text { Fadiga }\end{array}$ & $\begin{array}{c}\text { Área de } \\
\text { Aço } \\
\text { Total }\end{array}$ & $\begin{array}{l}\text { Área de } \\
\text { Aço } \\
\text { Mínima }\end{array}$ & $\begin{array}{c}\text { Armadura } \\
\text { de } \\
\text { Reforço } \\
\text { Necessária }\end{array}$ \\
\hline $\begin{array}{c}\mathrm{V}_{\mathrm{d}, \text { serv. máx. }} \\
(\mathrm{kN})\end{array}$ & $\begin{array}{l}V_{d, \text { serv. min. }} \\
(\mathrm{kN})\end{array}$ & $\begin{array}{c}\sigma_{1} \\
\left(\mathrm{kN} / \mathrm{cm}^{2}\right)\end{array}$ & $\begin{array}{c}\sigma_{2} \\
\left(\mathrm{kN} / \mathrm{cm}^{2}\right)\end{array}$ & $\begin{array}{c}\Delta \sigma_{\mathrm{w}} \\
\left(\mathrm{kN} / \mathrm{cm}^{2}\right)\end{array}$ & $\mathrm{ff}$ & $\begin{array}{l}A_{\mathrm{sw}, \text { total }} \\
\left(\mathrm{cm}^{2} / \mathrm{m}\right)\end{array}$ & $\begin{array}{c}\text { Asw,min. } \\
\left(\mathrm{cm}^{2} / \mathrm{m}\right)\end{array}$ & $\begin{array}{l}\text { Asw,ref. } \\
\left(\mathrm{cm}^{2} / \mathrm{m}\right)\end{array}$ \\
\hline 1.395 & 768 & 213 & 52,6 & 160 & 1,885 & 54,5 & 11,4 & 37,7 \\
\hline
\end{tabular}

\section{Discussão dos resultados}

A partir dos resultados dos dimensionamentos apresentados, são analisadas as condicionantes técnicas e construtivas. Para o reforço com encamisamento em concreto armado, faz-se a comparação entre as solicitações características e de dimensionamento das seções de cálculo à direita do apoio $\left(\mathrm{S}_{0}\right)$ para a força cortante e de meio de vão $\left(S_{5}\right)$ para o momento de flexão das longarinas do tabuleiro das pontes, além de suas armaduras longitudinais e transversais.

No projeto de reabilitação foram incorporadas lajes de transição e consoles de apoio, portanto, surgem novos carregamentos gerados por estes elementos. Em relação às ações variáveis, as principais diferenças entre as normas estão associadas ao trem-tipo de projeto e ao coeficiente de impacto.

No encamisamento em concreto armado, os carregamentos de sobrecarga permanente e carga móvel permaneceram os mesmos daqueles apresentados no Quadro 1 e no Quadro 2 para a ponte reabilitada. Entretanto, observa-se o aumento do peso próprio da estrutura, sendo a principal desvantagem dessa técnica.

As variações entre os carregamentos da ponte reabilitada com aumento de seção e a ponte original são apresentadas no Quadro 7 e no Quadro 8. Conforme o DNIT (1996), as solicitações de carga permanente da laje de transição somente devem ser consideradas quando desfavorável para a estrutura. Portanto, são apresentados dois valores para as solicitações internas devidas à sobrecarga permanente, obtidos separando-se a laje de transição dos demais carregamentos.

As alterações dos carregamentos se traduzem em mudanças nas solicitações das pontes em estudo. Como pode ser observado no Quadro 7, a diferença entre os momentos de flexão devido ao peso próprio estrutural é de $27,6 \%$ e de $74,4 \%$ devido à sobrecarga permanente utilizada na combinação mínima de ações. A mudança do trem-tipo TB-360 para o TB-450 e o aumento dos coeficientes de impacto resultam em um aumento de $91,0 \%$ nos momentos de flexão. Os valores desses esforços solicitantes estão plotados no gráfico da Figura 7.

Em relação à força cortante, a variação entre as solicitações devidas ao peso próprio estrutural é de $25,7 \%$ e devidas à sobrecarga permanente é de $30,9 \%$. A variação da carga móvel resulta em um aumento de $139,1 \%$ da força cortante mínima, como indicado na Tabela 8. Os valores desses esforços solicitantes estão plotados no gráfico da Figura 8.

Reforço das Longarinas de uma Ponte em Concreto Armado por meio de Encamisamento em Concreto Armado 
Quadro 7 - Momentos de flexão característicos na seção $S_{5}$

\begin{tabular}{|c|c|c|c|}
\hline \multicolumn{4}{|c|}{ MOMENTO FLETOR CARACTERÍsTICO } \\
\hline Seção $S_{5}$ & Ponte Original & $\begin{array}{c}\text { Ponte Reabilitada com } \\
\text { Encamisamento }\end{array}$ & Variação \\
\hline$M_{g 1}(k N . m)$ & 1.371 & 1.749 & $27,6 \%$ \\
\hline \multirow{2}{*}{$M_{g 2}(k N . m)$} & 847 & $1.004 *$ & $18,6 \%$ \\
\hline$M_{q 1}(k N . m)$ & 2.660 & $217^{* *}$ & $-74,4 \%$ \\
\hline$M_{\mathrm{q} 2}(\mathrm{kN} . \mathrm{m})$ & -780 & 3.899 & $46,6 \%$ \\
\hline
\end{tabular}

* Valores máximos da envoltória; ** Valores mínimos da envoltória

Quadro 8 - Momentos de flexão característicos na seção $\mathrm{S}_{0, \mathrm{~d}}$

\begin{tabular}{|c|c|c|c|}
\hline \multicolumn{5}{|c|}{ FORÇAS CORTANTES CARACTERÍSTICAS } \\
\hline Seção So,d $^{-5}$ & Ponte Original & $\begin{array}{c}\text { Ponte Reabilitada com } \\
\text { Encamisamento }\end{array}$ & Variação \\
\hline$V_{\mathrm{g} 1}(\mathrm{kN})$ & 452 & 568 & $25,7 \%$ \\
\hline $\mathrm{V}_{\mathrm{g} 2}(\mathrm{kN})$ & 212 & 278 & $30,9 \%$ \\
\hline $\mathrm{V}_{\mathrm{q} 1}(\mathrm{kN})$ & 553 & 1.098 & $98,5 \%$ \\
\hline $\mathrm{V}_{\mathrm{q} 2}(\mathrm{kN})$ & $-64,5$ & -156 & $-139,1 \%$ \\
\hline
\end{tabular}

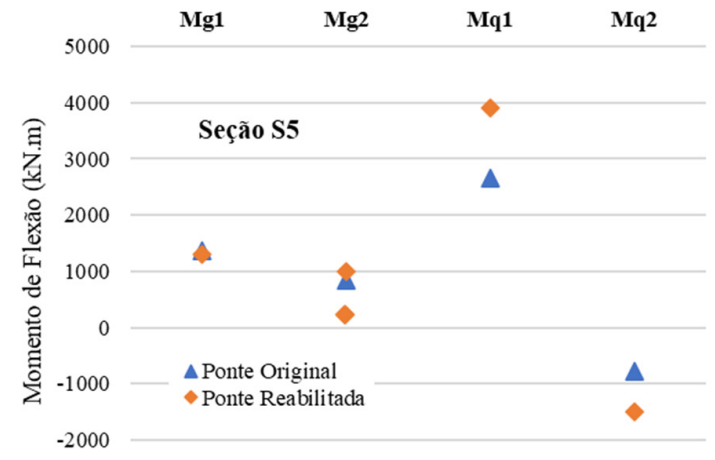

Figura 7 - Momentos de flexão característicos na seção $\mathrm{S}_{5}$

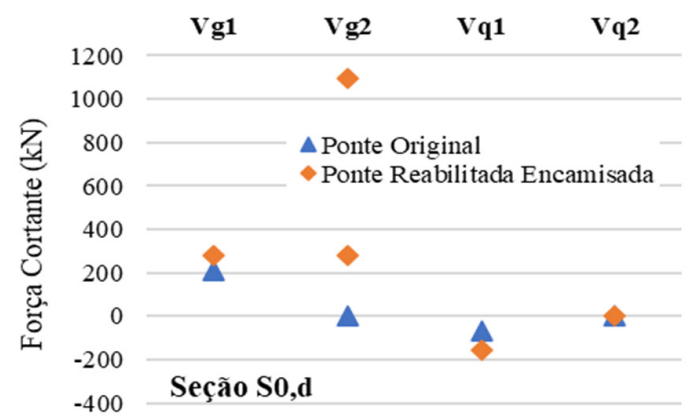

Figura 8 - Forças cortantes características na seção $\mathrm{S}_{0, \mathrm{~d}}$

As solicitações para o dimensionamento à flexão e ao cisalhamento são apresentadas no Quadro 9. A partir da análise dos resultados, observa-se um acréscimo de $26,7 \%$ na força cortante de dimensionamento e o surgimento de um momento de flexão negativo na combinação mínima de ações.

O Quadro 10 mostra as armaduras transversais e longitudinais da ponte original e do projeto de reabilitação por encamisamento. A variação da área de aço longitudinal inferior se deve pelo valor negativo do momento de flexão da combinação mínima de ações, o qual antes era nulo, e, portanto, o que correspondia a armadura montagem. No que se refere à armadura transversal, o aumento foi de $224 \%$. 
Quadro 9 - Solicitações de dimensionamento

\begin{tabular}{|c|c|c|c|}
\hline \multicolumn{4}{|c|}{ SOLICITAÇÕES DE DIMENSIONAMENTO } \\
\hline \multirow{2}{*}{ Projeto } & \multicolumn{2}{|c|}{ Momento de Flexão $\left(S_{5}\right)$} & \multirow{2}{*}{$\frac{\text { Força Cortante }\left(\mathrm{S}_{0, \mathrm{~d}}\right)}{\mathrm{V}_{\mathrm{sd}}(\mathrm{kN})}$} \\
\hline & $M_{s d, m a ́ x . ~}(k N . m)$ & $\mathrm{M}_{\mathrm{sd}, \mathrm{min} .}(\mathrm{kN} . \mathrm{m})$ & \\
\hline Ponte Original & 8.980 & 9.566 & 2.202 \\
\hline $\begin{array}{l}\text { Ponte Reabilitada com } \\
\text { Encamisamento }\end{array}$ & 657 & -270 & 2.789 \\
\hline Variação & $-92,7 \%$ & $-102,8 \%$ & $26,7 \%$ \\
\hline
\end{tabular}

Quadro 10 - Armaduras transversais e longitudinais

\begin{tabular}{|c|c|c|c|}
\hline \multicolumn{4}{|c|}{ ÁREAS DE AÇO } \\
\hline \multirow{2}{*}{ Projeto } & \multicolumn{2}{|c|}{ Armadura Longitudinal $\left(\mathbf{S}_{5}\right)$} & Armadura Transversal $\left(\mathrm{S}_{0, \mathrm{~d}}\right)$ \\
\hline & $A_{s, \text { inf. }}\left(\mathrm{cm}^{2}\right)$ & $A_{s, \text { sup }} \cdot\left(\mathrm{cm}^{2}\right)$ & $A_{s w}\left(\mathrm{~cm}^{2} / \mathrm{m}\right)$ \\
\hline Ponte Original & 2,45 & 129 & 16,8 \\
\hline $\begin{array}{c}\text { Ponte Reabilitada com } \\
\text { Encamisamento }\end{array}$ & 45,8 & 152 & 54,5 \\
\hline Variação & $1771 \%$ & $18,0 \%$ & $224 \%$ \\
\hline
\end{tabular}

\section{Conclusões}

A reabilitação, além de recuperar e reforçar a ponte, introduz modificações, tais como aumento da capacidade de carga, alargamento, adição de passeios laterais e barreiras de segurança. À vista disto, o reforço estrutural de estruturas tem sido uma alternativa para novas construções, possibilitando a realização de modificações em sua seção transversal e que resistam a cargas de projeto mais altas, além de corrigir a perda de resistência devido à deterioração.

O método de encamisamento em concreto armado é o mais utilizado para o reforço de pontes. A metodologia de cálculo do dimensiamento do reforço não apresenta variações daquela de um elemento estrutural novo em concreto armado. Por outro lado, o aumento da seção transversal gerou o aumento do peso próprio estrutural e, como consequência, observou-se um incremento de até $39,1 \%$ no momento de flexão no meio do vão da ponte reabilitada.

Apesar disso, o encamisamento em concreto armado se mostrou como uma solução para o reforço estrutural da ponte em estudo.

\section{Referências Bibliográficas}

Associação Brasileira de Normas Técnicas. ABNT NB-1 (1960): Cálculo e Execução de Obras em Concreto Armado. Rio de Janeiro.

Associação Brasileira de Normas Técnicas. ABNT NB-2 (1961): Cálculo e Execução de Pontes de Concreto Armado. Rio de Janeiro.

Associação Brasileira de Normas Técnicas. ABNT NB-6 (1960): Cargas Móveis em Pontes Rodoviárias. Rio de Janeiro.

Associação Brasileira de Normas Técnicas. ABNT NB-7 (1943): Cargas Móveis em Pontes Ferroviárias. Rio de Janeiro.

Reforço das Longarinas de uma Ponte em Concreto Armado por meio de Encamisamento em Concreto Armado 
Associação Brasileira de Normas Técnicas. ABNT NBR 6118 (2014): Projeto de Estruturas de Concreto Procedimento. Rio de Janeiro.

Associação Brasileira de Normas Técnicas. ABNT NBR 7480 (2007): Aço destinado a armaduras para estruturas de concreto armado - Especificação. Rio de Janeiro.

Associação Brasileira de Normas Técnicas. ABNT NBR 7187 (2003): Projeto de pontes de concreto armado e de concreto protendido - Procedimento. Rio de Janeiro.

Associação Brasileira de Normas Técnicas. ABNT NBR 7188 (2013): Carga móvel rodoviária e de pedestres em pontes, viadutos, passarelas e outras estruturas. Rio de Janeiro.

Associação Brasileira de Normas Técnicas. ABNT NBR 8681 (2004): Ações e segurança nas estruturas Procedimento. Rio de Janeiro.

Comité Euro-International du Béton. (1983) CEB Bulletin d'Information no.162 - Assessment of concrete structures and design procedures for upgrading (redesign). Lausanne, Suíça.

Comité Euro-International du Béton. (1993) CEB Bulletin d'Information no.213/214 - CEB-FIP Model Code 1990. Lausanne, Suíça.

Departamento Nacional de Infraestrutura de Transportes. DNIT (1996) Manual de Projeto de Obras de Arte Especiais. Rio de Janeiro.

Departamento Nacional de Infraestrutura de Transportes. DNIT (2010) Manual de Recuperação de Pontes e Viadutos Rodoviários. Rio de Janeiro.

Guimarães, R.S.. (2020) Reabilitação de Ponte em Concreto Armado com Encamisamento em Concreto Armado, Colagem de PRFC e de Chapas de Aço. Niterói. Projeto de Conclusão de Curso II. Departamento de Engenharia Civil. Universidade Federal Fluminense, Niterói.

Simões, M.L.. (2007) Reforço à Flexão de Vigas de Concreto Armado por Encamisamento Parcial. Rio de Janeiro: Universidade Federal do Rio de Janeiro.

Thomaz, E.C.S.. Cimentos e Concretos - 1900 a 2010. (2010) In: III Congresso Brasileiro de Pontes e Estruturas CBPE. Rio de Janeiro.

Reforço das Longarinas de uma Ponte em Concreto Armado por meio de Encamisamento em Concreto Armado 